\title{
Aplicación de instrumentos estandarizados de evaluación formativa y su influencia en el mejoramiento del desempeño docente Huaraz, 2018
}

\section{Application of standardized formative evaluation instruments and their influence on the improvement of teaching performance Huaraz, 2018}

(iD) 'Alberto Huamaní Gallo (iD 2Elías Jesús Mejía Mejía

Universidad Nacional Santiago Antúnez de Mayolo. Ancash, Perú.

Universidad Nacional Mayor de San Marcos. Lima, Perú. ${ }^{2}$

\section{Resumen}

El presente trabajo de investigación, está referido a la problemática que presentan los"docentes del Área de Arte del Nivel de Educación Secundaria, de la Educación Básica Regular de la ciudad de Huaraz y parte del Callejón de Huaylas 2018", donde los referidos profesionales presentan dificultades en elaborar y aplicar instrumentos estandarizados de evaluación formativa, para evaluar las actividades artísticas propias del área curricular; y cómo este factor influye en la baja calidad del desempeño docente. Frente a este problema, se planteó la intervención con un nivel de investigación experimental, constituyéndose dos grupos: grupo control y grupo experimental; y siguiendo el protocolo de la metodología de la investigación científica, se hizo la intervención al grupo experimental, con estímulos en la elaboración y aplicación de instrumentos estandarizados de evaluación formativa, para evaluar actividades artísticas musicales, danzarías, teatrales y artes visuales, en la que se pudo determinar, la influencia en el mejoramiento de la calidad del desempeño de docentes del grupo experimental. Al finalizar la intervención, los docentes participantes lograron significativas puntuaciones de mejora, como, excelente, muy bueno y bueno; frente al grupo control, que permaneció con calificativos de regular y deficiente; es decir, con baja calidad del desempeño docente, debido a que no han recibido el estímulo de mejora en la elaboración y aplicación de instrumentos estandarizados de evaluación formativa.

Palabras clave: Instrumentos de evaluación, instrumentos estandarizados de eva-

Recibido:

Marzo 2020

Aceptado: Junio 2020

luación, evaluación formativa, desempeño docente, calidad de desempeño docente.

\section{Abstract}

This research work is related to the problems presented by the "teachers of the Art Area of the Secondary Education Level, of the Regular Basic Education of the 
city of Huaraz and part of the Callejón de Huaylas 2018", where the aforementioned professionals present difficulties in developing and applying standardized formative assessment instruments to evaluate the artistic activities typical of the curricular area; and how this factor influences the low quality of teaching performance. Faced with this problem, the intervention was proposed with a level of experimental research, constituting two groups: control group and experimental group; and following the protocol of the scientific research methodology, the intervention was made to the experimental group, with stimuli in the elaboration and application of standardized instruments of formative evaluation, to evaluate musical artistic activities, dances, theatrical and visual arts, in which it was possible to determine, the influence in the improvement of the quality of the performance of teachers of the experimental group. At the end of the intervention, the participating teachers achieved significant improvement scores, such as, excellent, very good and good; compared to the control group, which remained classified as fair and poor; that is, with low quality of teaching performance, because they have not received the stimulus for improvement in the development and application of standardized instruments for formative evaluation.

Keywords: Evaluation instruments, standardized evaluation instruments, formative evaluation, teaching performance, quality of teaching performance.

\section{INTRODUCCIÓN}

El Arte como una actividad innata o innato del ser humano, es de suma importancia en el desarrollo integral de la persona de cualquier edad. Permite expresar sentimientos, estados de ánimo, formas de pensar, de actuar, mediante los distintos lenguajes artísticos como la música, la danza, el teatro, las artes visuales. El Ministerio de Educación (MINEDU), en el Diseño Curricular Nacional (DCN) de Educación Básica Regular (EBR), fundamenta que el Área de "Arte tiene como finalidad desarrollar en los estudiantes, la sensibilidad, la creatividad y el pensamiento crítico, para reconocer, valorar y apreciar las características de su cultura y de otras"; añade además que, "el arte, es la expresión propia, íntima y significativa de una persona y de un pueblo. (MED, DCN, 2009); "para expresar con libertad, sus percepciones, sus creencias, sus emociones y sentimientos; desarrollar su equilibrio afectivo, creativo y lúdico; y transformar su propio ser, para actuar con libertad, equidad y justicia" (Torres, 2013). En esta medida, el arte es medio por la cual, los estudiantes, exteriorizan sus emociones, sus frustraciones y preferen- cias; es un medio catártico de los estudiantes. El Arte, según Bunge (Bunge. 1999), significa una feliz conjunción de experiencia, destreza, imaginación, visión y habilidad, para realizar inferencias de tipo no analítico. Por lo tanto, el arte, la poesía, la religión, el amor, son experiencias de tipo no analítico, es decir experiencias subjetivas que afloran en el ser de manera innata y creativa, espontánea, como una manifestación de los estados de ánimo. Su campo de acción lo estudia la filosofía estética.

La evaluación de actividades artísticas en la EBR en el área de arte, no es tomado con especial atención por parte de los docentes encargados del desarrollo del área curricular, como agentes protagonistas del proceso educativo. El acto valorativo requiere de la intervención, mediante la aplicación sistemática de instrumentos de evaluación, en cada uno de los lenguajes artísticos. De tal forma que, el problema en cuestión es, la falta de aplicación sistemática, adecuada y apropiada de los instrumentos de evaluación, en el 
desarrollo de actividades artísticas de Artes Visuales, Teatro, Música y Danza; y esta deficiencia, influye definitivamente en la baja calidad del desempeño docente, del profesor del Área de Arte, en la Educación Básica Regular.

Desde distintos puntos de vista de la escuela, la educación y la sociedad en general, al arte le otorgan un papel de recreación, de expresión de libertad, como un área libre con pocos criterios valorativos. Así mismo, en ciertas instituciones educativas, el desarrollo del área de arte, se les encarga a docentes de otras áreas curriculares, como parte de relleno o complemento de su carga académica; para completar sus horas pedagógicas; acto totalmente negativo ocasionado por autoridades educativas. De modo que, al hacer la evaluación formativa, simplemente no cuentan con instrumentos apropiados, planificados. La evaluación no es sistemático, organizado, para cada lenguaje artístico. Este hecho trae como consecuencia la desvaloración del arte como área curricular; baja el nivel de interés por hacer arte por parte de los estudiantes; propicia que se desvalorice el arte; siendo este aspecto una debilidad en la calidad del desempeño docente, del profesional de educación artística.

En la evaluación de las actividades artísticas, en las instituciones educativas del nivel de educación secundaria de Huaraz, al no hacer uso de instrumentos de evaluación como herramienta importante, parte del proceso educativo, hay un sesgo hacia el subjetivismo; y este hecho, por una parte, afecta el real desenvolvimiento y desempeño de los estudiantes; $y$, por otra parte, afecta de manera negativa a la naturaleza del área de arte, al desvalorizar su esencia educativa. Simplemente no se involucra en el desarrollo de las capacidades y competencias de ciclo determinados en el currículo nacional de la EBR. Lo que resulta finalmente, una evaluación subjetiva y al azar, del desenvolvimiento de los estudiantes. Esta situación definitivamente determina la baja calidad del desempeño docente del Área de Arte.
En este contexto, la evaluación de los aprendizajes es un proceso continuo y sistemático, mediante el cual se observa, recoge, describe, procesa y analiza los logros, avances y/o dificultades del aprendizaje, con la finalidad de reflexionar, emitir juicios de valor y tomar decisiones oportunas y pertinentes para mejorar los procesos pedagógicos". MED (2005). Los Instrumentos de evaluación formativa, son medios físicos que permiten recoger o registrar información sobre el logro de aprendizaje y el desarrollo de competencias (MED, 2009).

En el Área de Arte, los instrumentos estandarizados de evaluación formativa que tienen significativa importancia, por ser muy prácticos, funcionales y confiables, para evaluar actividades artísticas, de los lenguajes artísticos de Artes Visuales, Teatro, Música y Danza, son de los siguientes: 1) Lista de cotejo: Individual y Grupal; 2) Guía de observación o ficha de observación; 3) Cuestionario; 4) Cuadro o ficha de progresión; 5) Ficha de evaluación de actividades de arte integral; 6) Ficha de evaluación de expresión dancística o de danza; 7) Ficha de registro de participantes en actividades de música, danza, teatro, canto; 8) Ficha mixta de evaluación artística grupal; 9) Ficha de evaluación de interpretación coral; 10) Ficha de evaluación de consolidado de ejecución artística; 11) Ficha de evaluación de actividades dramáticas ; 12) Ficha evaluación de ejecución teatral; 13) Rúbrica para evaluar actividades artísticas mixtas; 14) Rúbrica para evaluar una exposición (Clase teórica de arte); 15) Escala de estimación para evaluar actividades dramáticas; 16) Instrumentos estandarizados para evaluar contenidos actitudinales; 17) Instrumento estandarizado de evaluación para evaluar comportamiento y actitud frente al área.

Los citados instrumentos estandarizados de evaluación formativa, han sido elaborados de manera sistemática, planificada y organizada, con los profesores que conformaron el grupo experimental; luego han sido aplicados en sus instituciones educativas, logrando con la inves- 
tigación, resultados muy favorables y alentadoras, en la que se pudo contrastar mediante la aplicación de estos instrumentos, la mejora de la calidad del desempeño, de docentes del Área de Arte del Nivel de Educación Secundaria, de la Educación Básica Regular de la ciudad de Huaraz y parte del Callejón de Huaylas 2018.

\section{MATERIALES Y MÉTODOS}

Los métodos utilizados en el desarrollo de la presente investigación fueron:

1. El método científico. Mediante el cual se ha elegido una serie de procedimientos de tipo lógico y experimental que permitieron comprobar las hipótesis mediante experiencias controladas, replicables y precisas.

2. Los métodos lógicos y empíricos. Los métodos lógicos, implican la utilización del pensamiento y el razonamiento para ejecutar deducciones, análisis y síntesis; mientras que los métodos empíricos de investigación, permiten aproximarse al conocimiento, mediante experiencias replicables, controladas y documentadas, que se conocen como experimentos.

El estudio corresponde al Tipo Cuantitativo Causal, porque señala a las variables de investigación que están relacionadas y proporcionan un sentido de contenidos del fenómeno al que hace referencia, destacando los estudios sobre búsquedas de causas, para saber los efectos que producen. "Los estudios explicativos van más allá de la descripción de conceptos o fenómenos, es decir, están dirigidos a responder por las causas de los eventos y fenómenos físico o sociales, como su nombre lo indica, su interés se centra en explicar por qué ocurre un fenómeno y en qué condiciones se manifiesta" (Hernández S., Fernández C., \& Baptista L., 2016).

En educación los hechos son parte de la realidad problemática que requieren ser investigados, acción de la investigación que se traduce en la relación causa-efecto y exige la presencia de un grupo experimental y un grupo de control.
El nivel de la presente investigación es: Experimental, porque describe la relación entre las variables, en un momento determinado, en función de la relación causa - efecto (Hernández, 2010). En consecuencia, la presente investigación determina la aplicación de instrumentos estandarizados de evaluación formativa y su influencia en el mejoramiento de la calidad del desempeño de docentes de educación secundaria del Área de Arte de instituciones educativas de Huaraz, 2018.

El diseño que corresponde es el cuasi-experimental. En estos casos se asume que la estrategia que se aplica es 'cuasi' o muy próxima a la experimental. (Mejía, 2017); el término"cuasi-experimento" se refiere a diseños de investigación experimentales en los cuales los sujetos o grupos de sujetos de estudio no están asignados aleatoriamente (Rossi y Freeman, 1993). Lo que determina el grado de control que se impone a las variables que se están estudiando, permitiendo manipular a la Variable Independiente "Aplicación de instrumentos estandarizados de evaluación formativa" para ver los efectos o su influencia en relación con la Variable Dependiente "mejoramiento de la calidad del desempeño de docentes de educación secundaria del Área de Arte de instituciones educativas de Huaraz".

En este diseño los integrantes de los grupos Experimental y de Control, están constituidos antes de iniciar la investigación y por ello, el diseño considera la manipulación de la variable independiente, es decir solo del Grupo Experimental, mientras que, en el Grupo de Control, no se aplica ningún tratamiento en el periodo experimental.

Para probar la validez de las hipótesis, se recurrió a la aplicación de los estadísticos: Pruebas "F"; "t" de Student; Prueba de comparación de medias; Puntuación "Z"y la Diferencia de Medias de Wilcoxon. De tal manera que para el análisis de datos se empleó la prueba " $t$ " de Student, para muestras relacionales. Es una distribución 
de probabilidad que surge del problema de estimar la media de una población normalmente distribuida cuando el tamaño de la muestra es pequeño. La distribución " $t$ " de Student es la distribución de probabilidad del cociente (Supo, 2014). Para el procesamiento de datos se empleó el software SPSS v25.

La población estuvo conformado por docentes de educación artística, que laboran en las instituciones educativas del nivel de educación secundaria del ámbito de la Provincia de Huaraz y parte del Callejón de Huaylas, de Educación Básica Regular, en un promedio de 80 docentes de la zona urbana y rural, donde presentan características homogéneas, por estar vinculados en el desarrollo y aplicación de un modelo curricular nacional, con características mínimas de variación por la diversificación curricular.

El tipo de muestra seleccionada para el desarroIlo de la presente investigación fue de carácter probabilística, representativa y determinativa, debido a que, en las instituciones educativas seleccionadas, presentan características homogéneas; conformada por 64 docentes localizadas en el ámbito de la provincia de Huaraz y parte del callejón de Huaylas del nivel de Educación
Secundaria, de Educación Básica Regular. En este contexto se tuvo de entre 01 a 04 docentes por institución educativa, que tienen a cargo el desarrollo del Área de Arte, distribuidos en dos grupos: 32 docentes para el Grupo Experimental y otros 32 docentes para el Grupo Control respectivamente.

\section{RESULTADOS Y DISCUSIÓN}

Para realizar el trabajo de campo, se ha establecido cuantitativamente el grupo experimental 32 profesores y grupo control 32 profesores entre nombrados y contratados del área de Arte, del nivel de educación secundaria, de instituciones educativas del ámbito de la ciudad de Huaraz y parte del Callejón de Huaylas de la zona urbana y zona rural respectivamente.

Ambos grupos tienen a su cargo, estudiantes del 1ro. al 5to. grado de educación secundaria; con un diseño curricular nacional oficial, que varía en un 30\% de contenidos temáticos como producto de la diversificación curricular y adecuación al contexto geográfico, cultural, social del ámbito de la institución educativa. En ambos grupos antes de la intervención con los talleres, se aplicó la prueba de entrada el Pretest y al finalizar el taller la prueba de salida Postest, cuyos resultados se muestran a continuación.

\section{Presentación, análisis e interpretación de datos}

Tabla 1. Representación de la aplicación sistemática de instrumentos estandarizados de evaluación

\begin{tabular}{lllll}
\hline \multirow{2}{*}{ Indicador } & \multicolumn{2}{l}{ Grupo Control $(\mathbf{n}=\mathbf{3 2})$} & \multicolumn{2}{l}{ Grupo Experimental $(\mathbf{n}=\mathbf{3 2})$} \\
& Frecuencia & Porcentaje & Frecuencia & Porcentaje \\
\hline Peficiente & PRETEST & & 24 & 75.0 \\
Regular & 24 & 75.0 & 8 & 25.0 \\
Total & 8 & 25.0 & 32 & 100 \\
Media & 32 & 100 & 1.25 & \\
Desviación estándar & 1.25 & & 0.44 & 00.0 \\
& 0.44 & & 0 & 00.0 \\
Deficiente & POSTEST & & 0 & 25.0 \\
Regular & 24 & 75.0 & 8 & 75.0 \\
Bueno & 8 & 25.0 & 00.0 \\
Muy Bueno & 0 & 00.0 & 24 & 100 \\
Excelente & 0 & 00.0 & 0 & \\
Total & 0 & 00.0 & 32 & \\
Media & 32 & 100 & 3.75 & \\
Desviación estándar & 1.25 & & 0.44 & \\
& 0.44 & & & \\
\hline
\end{tabular}

Fuente: Test aplicado a los docentes, elaborado por el autor. 


\section{a) Análisis e interpretación de datos del Pretest}

En la Tabla 1, referente al Grupo Control, se observa que el $25 \%$ obtuvo el calificativo de Regular y un $75 \%$ el calificativo de Deficiente.

Del mismo modo, en el Grupo Experimental, el $75 \%$ obtuvo el calificativo de Deficiente; el $25 \%$ obtuvieron el calificativo de Regular. En ambos casos, tanto el Grupo Control y el Grupo Experimental obtienen el calificativo de Deficiente y Regular respectivamente. Razón por la que se afirma que los docentes del Grupo Control y Grupo Experimental, no aplican sistemáticamente instrumentos estandarizados de evaluación formativa y que tienen baja calidad de desempeño docente en el Área de Arte, en Educación Secundaria de la ciudad de Huaraz, 2018.

\section{b) Análisis e interpretación de datos del Postest}

En la Tabla 1 se muestra que el $75 \%$ do- centes del Grupo Control obtuvieron el calificativo de Deficiente; y el $25 \%$ obtiene el calificativo de Regular. Quiere decir que mantienen su calificativo de deficiente y regular respectivamente.

Mientras que, en el Grupo Experimental, el $25 \%$ de la muestra, obtienen el calificativo de Bueno; y el $75 \%$ tienen el calificativo de Muy Bueno. En este caso, se observa que el Grupo Experimental ha mejorado significativamente su calificativo posterior a haber recibido el estímulo; mientras que el Grupo Control mantiene su calificativo de deficiente y regular, como consecuencia de no haber recibido el estímulo. Razón por la que se afirma que los docentes del Grupo Experimental, si aplican sistemáticamente instrumentos estandarizados de evaluación formativa como parte de la calidad de desempeño docente en el Área de Arte, en Educación Secundaria de la ciudad de Huaraz, 2018.

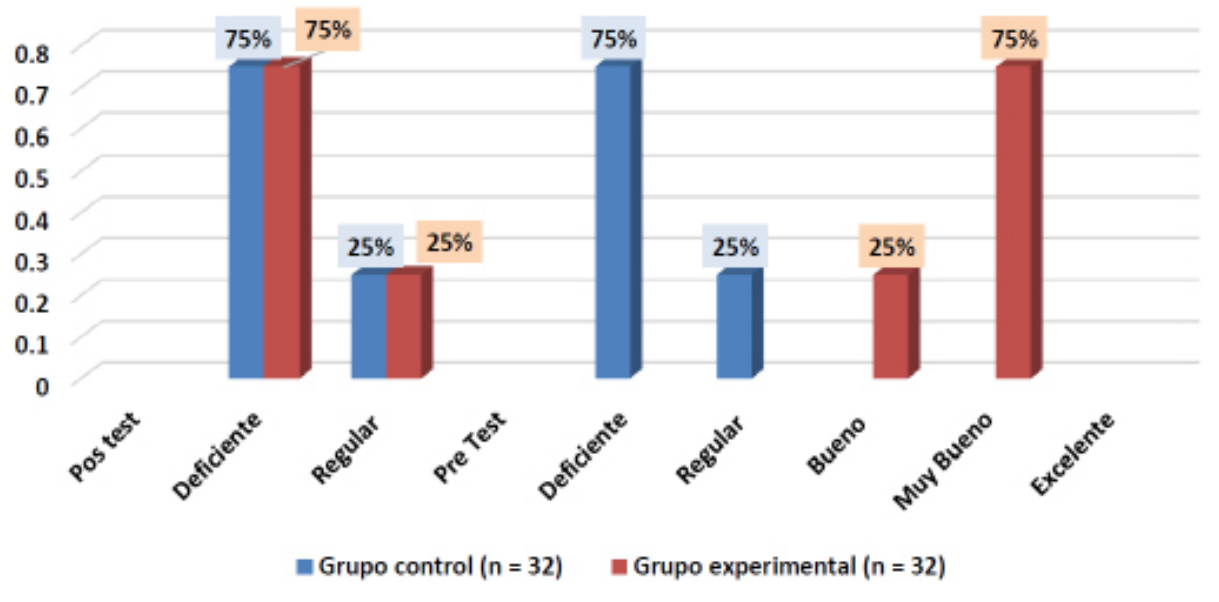

Figura 1. Aplicación sistemática de instrumentos estandarizados de evaluación formativa 
Tabla 2. Representación de la evaluación sistemática de criterios, capacidades y del nivel de progreso de las competencias del área de arte.

\begin{tabular}{|c|c|c|c|c|}
\hline \multirow{2}{*}{ Indicador } & \multicolumn{2}{|c|}{ Grupo Control $(\mathrm{n}=32)$} & \multicolumn{2}{|c|}{ Grupo Experimental $(n=32)$} \\
\hline & Frecuencia & Porcentaje & Frecuencia & Porcentaje \\
\hline & \multicolumn{4}{|c|}{ PRETEST } \\
\hline Deficiente & 20 & 62.5 & 20 & 62.5 \\
\hline Regular & 12 & 37.5 & 12 & 37.5 \\
\hline Total & 32 & 100 & 32 & 100 \\
\hline Media & \multicolumn{2}{|c|}{1.38} & \multicolumn{2}{|c|}{1.38} \\
\hline \multirow[t]{2}{*}{ Desviación estándar } & \multicolumn{2}{|c|}{0.492} & \multicolumn{2}{|c|}{0.492} \\
\hline & \multicolumn{4}{|c|}{ POSTEST } \\
\hline Deficiente & 20 & 62.5 & 0 & 00.0 \\
\hline Regular & 12 & 37.5 & 0 & 00.0 \\
\hline Bueno & 0 & 00.0 & 12 & 37.5 \\
\hline Muy Bueno & 0 & 00.0 & 8 & 25.0 \\
\hline Excelente & 0 & 00.0 & 12 & 37.5 \\
\hline Total & 32 & 100 & 32 & 100 \\
\hline Media & \multicolumn{2}{|c|}{1.38} & \multicolumn{2}{|c|}{4.00} \\
\hline Desviación estándar & \multicolumn{2}{|c|}{0.492} & \multicolumn{2}{|c|}{0.88} \\
\hline
\end{tabular}

Fuente: Test aplicado a los docentes, elaborado por el autor.

\section{a) Análisis e interpretación de datos del PRETEST}

En la Tabla 2 se observa el $62.5 \%$ de docentes del Grupo Control obtuvieron el calificativo de Deficiente; y el 37.5 \% obtuvieron el calificativo de Regular.

Del mismo modo, se observa que el $62.5 \%$ de docentes del Grupo Experimental obtuvieron el calificativo de Deficiente; y el 37.5 \% obtuvieron el calificativo de Regular. En ambos casos, tanto el Grupo Control y el Grupo Experimental presentan el calificativo de Deficiente y Regular respectivamente.

\section{b) Análisis e interpretación de datos del Postest:}

En la Tabla 2 se observa que el $62.5 \%$ docentes del Grupo Control obtuvieron el calificativo de Deficiente; el $37.5 \%$ de la muestra obtuvieron el calificativo de Regular. Quiere decir que mantienen su calificativo de deficiente y regular respectivamente.

Del mismo modo observa que $37.5 \%$ de docentes del Grupo Experimental obtienen el calificativo de Bueno; el 25 \% de la muestra, obtienen el calificativo de Muy Bueno; y finalmente el 37.5 \% obtienen el calificativo de Excelente. En este caso, se observa que el Grupo Experimental ha mejorado significativamente su desempeño como consecuencia de haber recibido el tratamiento del taller.

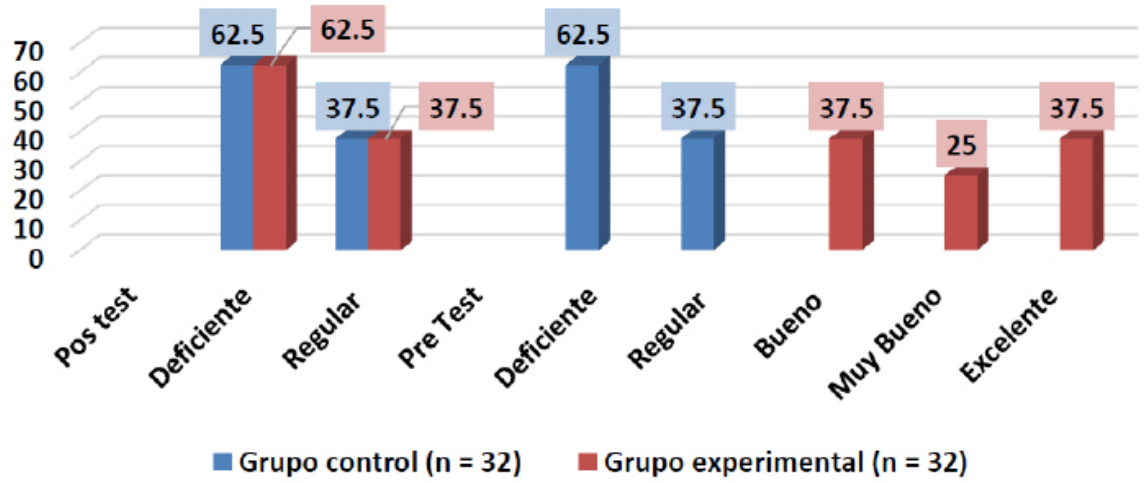

Figura 2. Representación de la evaluación sistemática de criterios, capacidades y del nivel de progreso de las competencias del área de arte. 
Tabla 3. Representación del uso de instrumentos estandarizados de evaluación formativa

\begin{tabular}{|c|c|c|c|c|}
\hline \multirow{2}{*}{ Indicador } & \multicolumn{2}{|c|}{ Grupo Control $(\mathrm{n}=32)$} & \multicolumn{2}{|c|}{ Grupo Experimental $(\mathrm{n}=32)$} \\
\hline & Frecuencia & Porcentaje & Frecuencia & Porcentaje \\
\hline & \multicolumn{4}{|c|}{ PRETEST } \\
\hline Deficiente & 28 & 87.5 & 28 & 87.5 \\
\hline Regular & 4 & 12.5 & 4 & 12.5 \\
\hline Total & 32 & 100 & 32 & 100 \\
\hline Media & \multicolumn{2}{|c|}{1.13} & \multicolumn{2}{|r|}{1.13} \\
\hline \multirow[t]{2}{*}{ Desviación estándar } & \multirow{2}{*}{\multicolumn{2}{|c|}{0.336}} & \multirow{2}{*}{\multicolumn{2}{|c|}{ POSTEST }} \\
\hline & & & & \\
\hline Deficiente & 28 & 87.5 & 0 & 00.0 \\
\hline Regular & 4 & 12.5 & 0 & 00.0 \\
\hline Bueno & 0 & 00.0 & 0 & 00.0 \\
\hline Muy Bueno & 0 & 00.0 & 16 & 50.0 \\
\hline Excelente & 0 & 00.0 & 16 & 50.0 \\
\hline Total & 32 & 100 & 32 & 100 \\
\hline Media & \multirow{2}{*}{\multicolumn{2}{|c|}{$\begin{array}{c}1.13 \\
0.336\end{array}$}} & \multirow{2}{*}{\multicolumn{2}{|c|}{$\begin{array}{c}4.50 \\
0.508\end{array}$}} \\
\hline Desviación estándar & & & & \\
\hline
\end{tabular}

Fuente: Test aplicado a los docentes, elaborado por el autor.

\section{a) Análisis e interpretación de datos del Pretest}

En la Tabla 3 se observa que el $87.5 \%$ de docentes del Grupo Control obtuvieron el calificativo de Deficiente; y el $12.5 \%$ obtiene el calificativo de Regular.

Del mismo modo, se observa que el $87.5 \%$ de docentes del Grupo Experimental, obtienen el calificativo de Deficiente; y el 12.5 \% de obtienen el calificativo de Regular. En ambos casos, tanto el Grupo Control y el Grupo Cuasi-Experimental presentan el calificativo de Deficiente y Regular respectivamente.

\section{b) Análisis e interpretación de datos del Postest}

En la Tabla 3 se observa que $87.5 \%$ de docentes del Grupo Control obtuvieron el calificativo de Deficiente; y el 12.5 \% obtuvieron el calificativo de Regular. Quiere decir que mantienen su calificativo de deficiente y regular.

Del mismo domo se observa que el $50 \%$ de docentes del Grupo Experimental obtienen el calificativo de Muy Bueno; y el 50 \% obtienen el calificativo de Excelente. En este caso, se observa que el Grupo Experimental ha mejorado significativamente su calificativo como consecuencia de haber recibido el estímulo. 


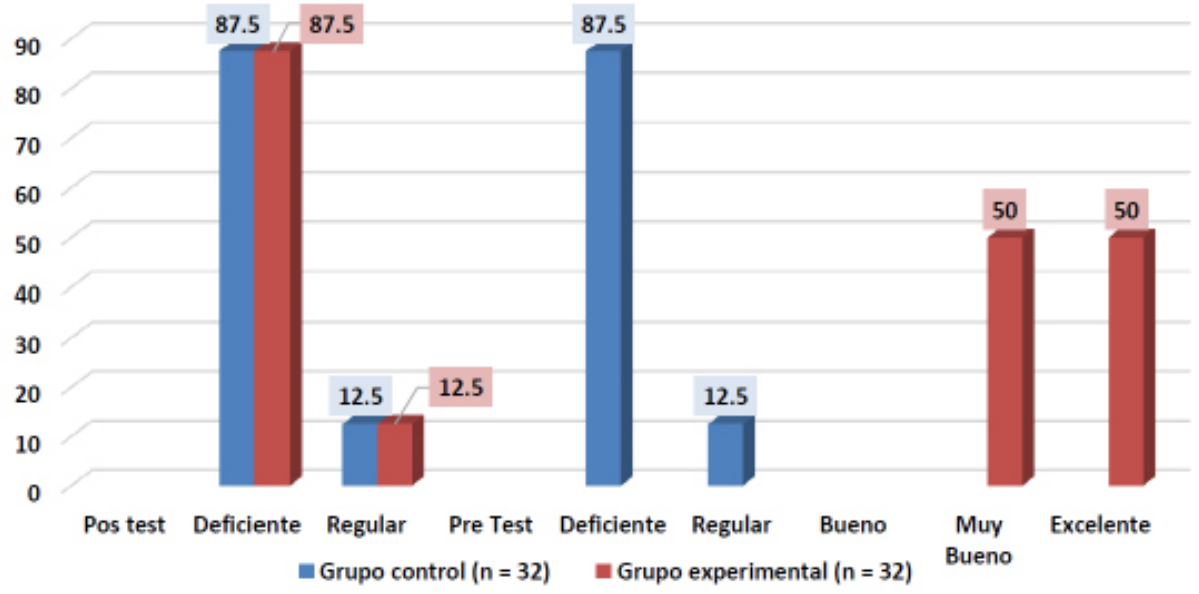

Figura 3. Representación gráfica del uso de instrumentos estandarizados de evaluación formativa

Tabla 4. Representación de la planificación y elaboración responsable de instrumentos de evaluación

\begin{tabular}{|c|c|c|c|c|}
\hline \multirow{2}{*}{ Indicador } & \multicolumn{2}{|c|}{ Grupo Control $(n=32)$} & \multicolumn{2}{|c|}{ Grupo Experimental $(n=32)$} \\
\hline & Frecuencia & Porcentaje & Frecuencia & Porcentaje \\
\hline & \multicolumn{4}{|c|}{ PRETEST } \\
\hline Deficiente & 28 & 87.5 & 28 & 87.5 \\
\hline Regular & 4 & 12.5 & 4 & 12.5 \\
\hline Total & 32 & 100 & 32 & 100 \\
\hline Media & \multicolumn{2}{|c|}{1.13} & \multicolumn{2}{|c|}{1.13} \\
\hline \multirow[t]{2}{*}{ Desviación estándar } & \multicolumn{2}{|c|}{0.336} & \multicolumn{2}{|c|}{0.336} \\
\hline & & & OSTEST & \\
\hline Deficiente & 28 & 87.5 & 0 & 00.0 \\
\hline Regular & 4 & 12.5 & 0 & 00.0 \\
\hline Bueno & 0 & 00.0 & 4 & 12.5 \\
\hline Muy Bueno & 0 & 00.0 & 23 & 71.9 \\
\hline Excelente & 0 & 00.0 & 5 & 15.6 \\
\hline Total & 32 & 100 & 32 & 100 \\
\hline Media & \multicolumn{2}{|c|}{1.13} & \multicolumn{2}{|c|}{4.03} \\
\hline Desviación estándar & \multicolumn{2}{|c|}{0.336} & \multicolumn{2}{|c|}{0.538} \\
\hline
\end{tabular}

Fuente: Test aplicado a los docentes, elaborado por el autor.

\section{a)Análisis e interpretación de datos del Pretest}

En la Tabla 4 se observa que el $87.5 \%$ de docentes del Grupo Control obtuvieron el calificativo de Deficiente; y el $12.5 \%$ obtiene el calificativo de Regular.

Del mismo modo, se observa que el $87.5 \%$ de docentes del Grupo Experimental obtienen el calificativo de Deficiente; y el $12.5 \%$ el calificativo de Regular. En ambos casos, presentan el calificativo de Deficiente y Regular respectivamente.

\section{b) Análisis e interpretación de datos del Postest}

En la Tabla 4 se observa que el $87.5 \%$ de docentes del Grupo Control obtuvieron el calificativo de Deficiente; y el $12.5 \%$ obtiene el calificativo de Regular. Quiere decir que mantienen su calificativo de deficiente y regular respectivamente.

Del mismo modo se observa que el $12.5 \%$ de docentes del Grupo Experimental obtienen 
el calificativo de Bueno; el $71 \%$ obtienen el calificativo de Muy Bueno y el $15.6 \%$ el calificativo de Excelente. En este caso, se observa que el Grupo Experimental ha mejorado significativamente su puntuación como consecuencia de haber recibido el estímulo.

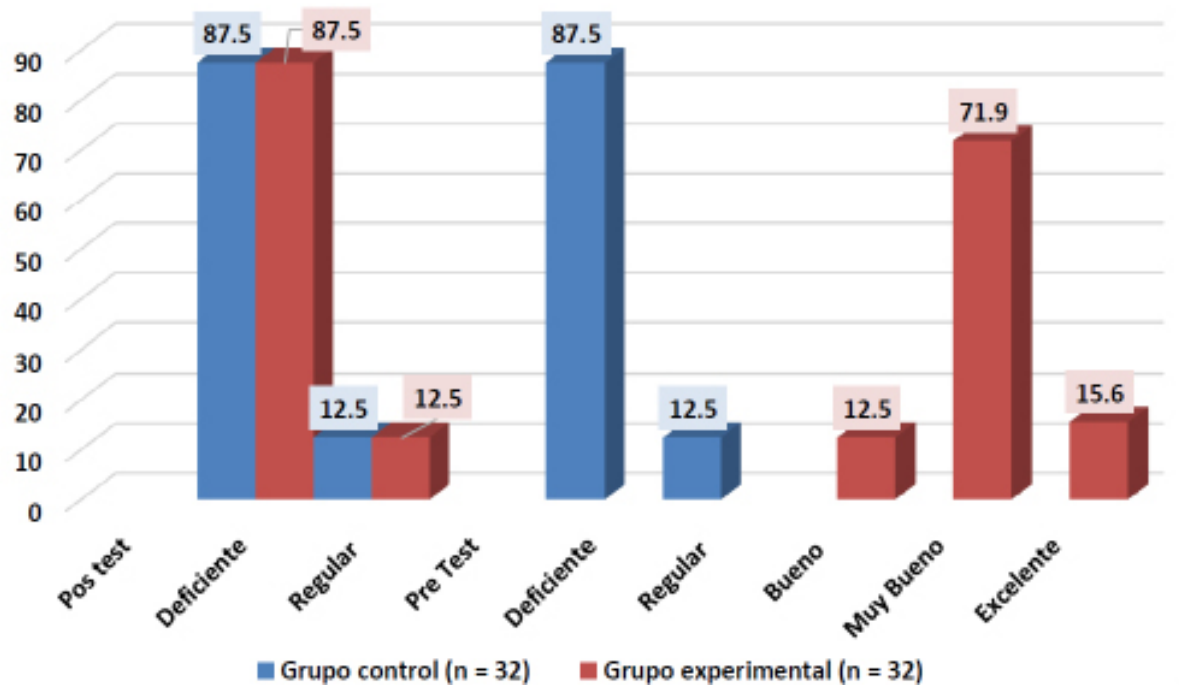

Figura 4. Representación gráfica de la planificación y elaboración responsable de instrumentos de evaluación.

Tabla 5. Representación de la calidad del desempeño docente

\begin{tabular}{|c|c|c|c|c|}
\hline \multirow{2}{*}{ Indicador } & \multicolumn{2}{|c|}{ Grupo Control $(n=32)$} & \multicolumn{2}{|c|}{ Grupo Experimental $(n=32)$} \\
\hline & Frecuencia & Porcentaje & Frecuencia & Porcentaje \\
\hline & \multicolumn{4}{|c|}{ PRETEST } \\
\hline Deficiente & 20 & 62.5 & 24 & 75.0 \\
\hline Regular & 12 & 37.5 & 8 & 25.0 \\
\hline Total & 32 & 100 & 32 & 100 \\
\hline Media & \multicolumn{2}{|c|}{1.38} & \multicolumn{2}{|r|}{1.25} \\
\hline \multirow[t]{2}{*}{ Desviación estándar } & \multirow{2}{*}{\multicolumn{2}{|c|}{0.492}} & \multicolumn{2}{|r|}{0.44} \\
\hline & & & POSTEST & \\
\hline Deficiente & 18 & 56.5 & 0 & 00.0 \\
\hline Regular & 14 & 43.8 & 0 & 00.0 \\
\hline Bueno & 0 & 00.0 & 12 & 37.5 \\
\hline Muy Bueno & 0 & 00.0 & 12 & 37.5 \\
\hline Excelente & 0 & 00.0 & 8 & 25.0 \\
\hline Total & 32 & 100 & 32 & 100 \\
\hline Media & \multicolumn{2}{|c|}{1.44} & \multicolumn{2}{|r|}{3.88} \\
\hline Desviación estándar & \multicolumn{2}{|c|}{0.504} & \multicolumn{2}{|r|}{0.793} \\
\hline
\end{tabular}

Fuente: Test aplicado a los docentes, elaborado por el autor.

\section{a) Análisis e interpretación de datos del \\ Pretest}

En la Tabla 5 se observa que el $62.5 \%$ de docentes del Grupo Control obtuvieron el ca-

lificativo de Deficiente; y 12 el $37.5 \%$ de la muestra obtienen el calificativo de Regular. 
Del mismo modo, se observa que el $75 \%$ de docentes del Grupo Experimental obtuvieron el calificativo de Deficiente; y el $25 \%$ obtiene el calificativo de Regular. En ambos casos, presentan el calificativo de Deficiente y Regular respectivamente.

\section{b) Análisis e interpretación de datos del Postest}

En la Tabla 5 se observa que el $56.5 \%$ de docentes obtuvieron el calificativo de Deficiente; y el $43.8 \%$ de la muestra obtuvieron el calificativo de Regular. Quiere decir que mantienen su calificativo de deficiente y regular respectivamente.

Del mismo domo se observa que el $37.5 \%$ de docentes del Grupo Experimental obtienen el calificativo de Bueno; el $75.5 \%$ obtienen el calificativo de Muy Bueno y el $25 \%$ el calificativo de Excelente. En este caso, se observa que el Grupo Experimental ha mejorado significativamente su calificativo como consecuencia de haber recibido el estímulo.

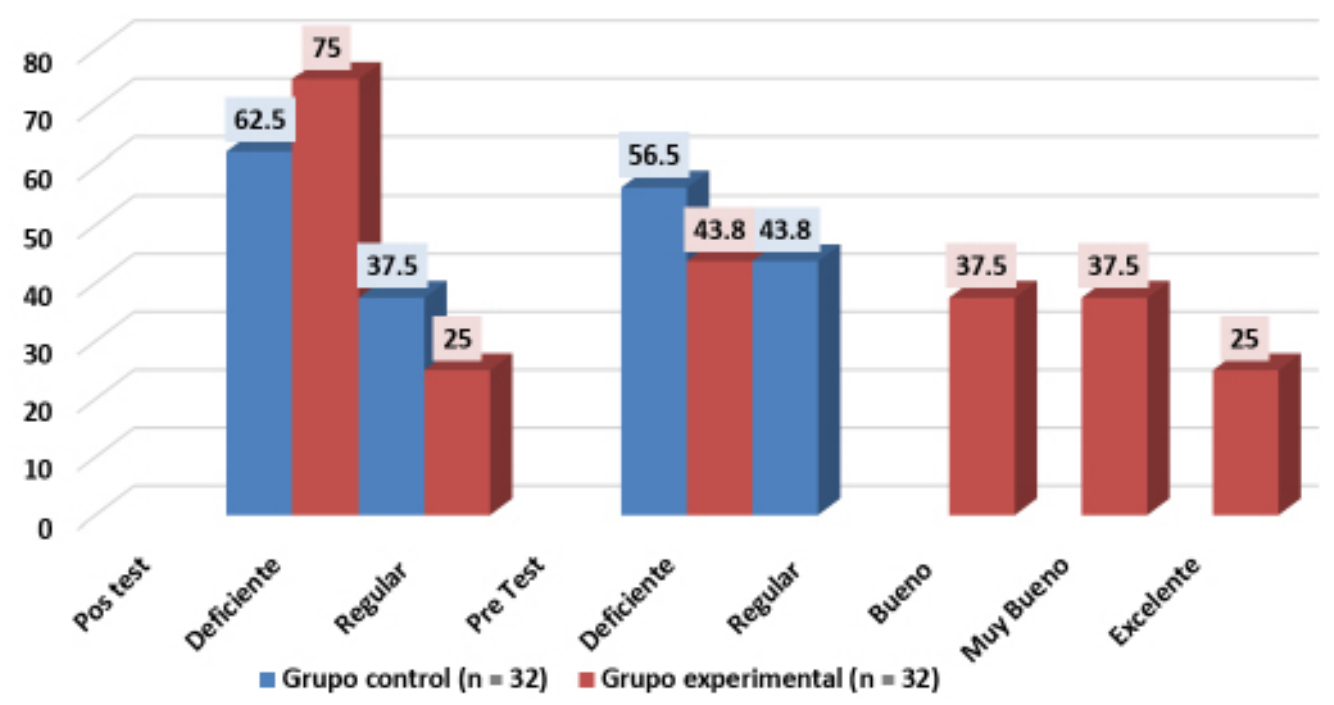

Figura 5. Representación gráfica de la calidad del desempeño docente

\section{1) Proceso de prueba de hipótesis}

El proceso de la Prueba de Hipótesis se hizo mediante la Prueba de Comparación de Medias para muestras dependientes; así mismo, se aplicó la prueba t de Student, si en ambos grupos se cumple el supuesto de normalidad; y en caso contrario, es decir, cuando no se cumple el supuesto de normalidad en uno o ambos grupos, para este caso, se aplicó la Prueba de Wilcoxon (Supo, 2014). 
Tabla 6. Representación de normalidad de los datos de Wilcoxon

\begin{tabular}{|c|c|c|c|c|}
\hline \multicolumn{5}{|c|}{ "PRUEBA DE NORMALIDAD DE LOS DATOS } \\
\hline & \multicolumn{3}{|c|}{ Kolmogorov-Smirnova } & \multirow[b]{2}{*}{ Prueba } \\
\hline & Estadístico & gl & Sig. & \\
\hline $\begin{array}{l}\text { Aplicación sistemática de los instrumentos } \\
\text { estandarizados de evaluación formativa Pretest }\end{array}$ &, 465 & 32 &, 000 & Wilcoxon \\
\hline $\begin{array}{l}\text { Evaluación sistemática de criterios, capacidades y } \\
\text { competencias del área que utilizan los docentes, en la } \\
\text { evaluación de actividades artísticas Pretest }\end{array}$ & ,402 & 32 & 000 & Wilcoxon \\
\hline $\begin{array}{l}\text { Instrumentos estandarizados de evaluación formativa } \\
\text { que utilizan los docentes en la evaluación de } \\
\text { actividades artísticas Pretest }\end{array}$ &, 520 & 32 & 000 & Wilcoxon \\
\hline $\begin{array}{l}\text { Planificación y elaboración responsable de } \\
\text { instrumentos estandarizados de evaluación formativa } \\
\text { para cada actividad artística Pretest }\end{array}$ &, 520 & 32 & 000 & Wilcoxon \\
\hline $\begin{array}{l}\text { Calidad del desempeño docente en el área de arte en } \\
\text { educación secundaria referido por estudiantes Pretest }\end{array}$ & ,402 & 32 & ,000 & Wilcoxon \\
\hline $\begin{array}{l}\text { Aplicación sistemática de los instrumentos } \\
\text { estandarizados de evaluación formativa Postest }\end{array}$ &, 465 & 32 &, 000 & Wilcoxon \\
\hline $\begin{array}{l}\text { Evaluación sistemática de criterios, capacidades y } \\
\text { competencias del área que utilizan los docentes, en la } \\
\text { evaluación de actividades artísticas Postest }\end{array}$ & ,402 & 32 &, 000 & Wilcoxon \\
\hline $\begin{array}{l}\text { Instrumentos estandarizados de evaluación formativa } \\
\text { que utilizan los docentes en la evaluación de } \\
\text { actividades artísticas Postest }\end{array}$ &, 520 & 32 &, 000 & Wilcoxon \\
\hline $\begin{array}{l}\text { Planificación y elaboración responsable de } \\
\text { instrumentos estandarizados de evaluación formativa } \\
\text { para cada actividad artística Postest }\end{array}$ &, 520 & 32 & ,000 & Wilcoxon \\
\hline $\begin{array}{l}\text { Calidad del desempeño docente en el área de arte en } \\
\text { educación secundaria referido por estudiantes Postest }\end{array}$ & 370 & 32 & ,000 & Wilcoxon' \\
\hline
\end{tabular}

Fuente: Base de datos del test aplicado a los docentes, elaborado por el autor.

\section{a) Análisis e interpretación}

Según se observa los resultados en tabla de representación de normalidad de los datos de Wilcoxon, donde el nivel de significancia es menor que $5 \%$; por lo tanto, se aplica la prueba de Wilcoxon para el caso de antes y después; y así demostrar que el estímulo dado al Grupo Experimental, es el conveniente.

\section{DISCUSIÓN}

Definitivamente existe gran preocupación por la poca responsabilidad e importancia que dan los docentes del área de arte de las instituciones educativas de la ciudad de Huaraz y parte del Callejón de Huaylas, a la planificación, elaboración y aplicación responsable de instrumentos estandarizados de evaluación formativa, para evaluar las distintas actividades artísticas: artes visuales, arte dramático, danza y música; que se desarrollan en la EBR como parte de los contenidos curriculares en la formación integral del estudiante. Cada lenguaje artístico aporta al desarrollo de las habilidades creativas, emocionales, desarrollo de la personalidad, la formación en valores y la responsabilidad del estudiante. Por lo que se requiere de una evaluación sistemática, planificada, organizada; menos subjetiva e improvisada. Toda actividad artística realizada en el salón de clases o al aire libre, debe tener mínimo un instrumento de evaluación, que realmente evidencie el aprendizaje del estudiante; una evaluación sistemática que tenga en cuenta los estándares de aprendizaje (criterios), las capacidades y por último el logro de las competencias previstas en el currículo nacional de la EBR, lo que, en definitiva, favorezcan cada vez el valor formativo del área de arte.

Definitivamente, el descuido de la función docente es tomado en cuenta como un in- 
dicador de baja calidad del desempeño docente; y lo contrario, el cumplimiento de las actividades de planificación, elaboración y aplicación responsable de instrumentos de evaluación formativa, obedecen a los estándares de calidad del desempeño del docente del área de arte.

El objetivo del presente trabajo fue: Demostrar que la aplicación sistemática de instrumentos estandarizados de evaluación formativa es un factor que influye en el mejoramiento de la calidad del desempeño de docentes del área de arte de instituciones educativas de la ciudad de Huaraz, 2018; y como resultado del estudio, mediante el apoyo de los estadísticos: Pruebas " $F$ "; " $t$ " de Student; Regresión Simple; Puntuación "Z" y la Diferencia de Medias de Wilcoxon respectivamente, se ha tenido como "valor de significación observada $p=$ 0.003, lo que es menor al valor de significación teórica $a=0.05$, por lo tanto, se rechaza la hipótesis nula y se acepta la hipótesis de investigación, que: "La aplicación sistemática de instrumentos estandarizados de evaluación formativa influye en el mejoramiento de la calidad del desempeño de docentes de educación secundaria del área de Arte de Instituciones Educativas de Huaraz, 2018".

Con lo cual se determinar que "la aplicación sistemática de instrumentos estandarizados de evaluación formativa, influyen en el mejoramiento de la calidad del desempeño docente del Área de Arte, en Educación Secundaria de Huaraz - 2018".

En consecuencia, la aplicación sistemática de instrumentos estandarizados de evaluación formativa, no está siendo tomada con responsabilidad, en el proceso pedagógico, como así lo afirma María José García San Pedro (2010), en su tesis doctoral concluye que: "Los instrumentos de evaluación, como herramientas de trabajo evaluativo, en el contexto educativo, no han sido desarrollados, es decir, los docentes no hacen uso en la prácti- ca educativa; durante el estudio realizado se evidenció que solo un docente de la muestra, hace uso de mapas de evaluación; el resto no dispone o no lo hace, lo que dificulta la visibilidad de la evaluación".

En el presente trabajo se ha podido contrastar la afirmación de García San Pedro (2010), "que, de una población determinada de docentes, más del $50 \%$ de docentes, se olvidan que tienen que hacer uso de instrumentos de evaluación, para recoger información de los logros de aprendizaje de los estudiantes", es decir, de lo que se ha programado, de lo que se ha planificado en las unidades didácticas y la programación curricular anual del curso. El dejar de hacer uso de un instrumento de evaluación formativa, hace que se pierda el interés, la debida importancia al área de arte; en ocasiones es considerado como área de recreación de los estudiantes para matar el tiempo libre, sin valores de evaluación y desarrollo de competencias.

\section{CONCLUSIONES}

Como parte final y conclusiva del presente trabajo de investigación, se toma como base, los resultados obtenidos y la discusión presentada, a partir de los datos empíricos estudiados, a la que formulan las siguientes conclusiones:

1. La aplicación sistemática de instrumentos estandarizados de evaluación formativa es un factor que influye significativamente en el mejoramiento de la calidad del desempeño de docentes de educación secundaria del área de arte de Instituciones Educativas de Huaraz, 2018. Conclusión que se arriba en base a los resultados de la prueba del estadístico t de Student, donde el valor de significación observada $p=0.003$ es menor al valor de significación teórica $a=0.05$.

2. En absoluto, la evaluación sistemática de criterios, capacidades y competencias del área que utilizan los docentes, en la evaluación de actividades artísticas influyen 
de manera positiva en la calidad del desempeño docente del área de arte, en educación secundaria de la ciudad de Huaraz. Conclusión que se adopta en base a los resultados mostrados de la prueba del estadístico t de Student, el valor de significación observada $p=0.000$ es menor al valor de significación teórica $a=0.05$.

3. Los instrumentos estandarizados de evaluación formativa que utilizan los docentes en la evaluación de actividades artísticas influyen significativamente en la calidad del desempeño docente del área de arte, en educación secundaria de la ciudad de Huaraz. Conclusión que se arriba en base a los resultados de la prueba del estadístico t de Student, el valor de significación observada $p=0.000$ es menor al valor de significación teórica $a=0.05$.

4. La planificación y elaboración responsable de instrumentos estandarizados de evaluación formativa para cada lenguaje artístico, Artes Visuales, Arte Dramático, Danza y Música; influye positivamente en la calidad del desempeño docente en el Área de Arte en educación secundaria de la ciudad de Huaraz. Conclusión que se arriba en base a los resultados de la prueba del estadístico t de Student, el valor de significación observada $p=0.017$ es menor al valor de significación teórica $a=0.05$.

\section{REFERENCIAS BIBLIOGRÁFICAS}

Bordas, M. I. (2001). Estrategias de evaluación de los aprendizajes centrados en el proceso. Barcelona: Universidad de Barcelona.

Bunge, M. (1997). La ciencia, su método y su fiIosofía. Buenos Aires: Sudamericana.

Coll, C. \&. (1993). La evaluación de los aprendizajes en el currículum escolar: Una perpectica constructivista. Madrid: s.e.

Hernández, Roberto; Fernández Carlos; Baptista, María (2016). Metodología de la investigación científica. México: Mexicana.
Hernández, R. (2010). Metodología de la investigación científica 5ta. Edición. México: Mc Graw Hill.

Ibarra, L. M. (2005). Desempeño Docente. Bogotá: Ministerio de Educación Nacional de Colombia.

Mejía M., E. J. (2017). La investigación científica en educación. Lima: Ediciones APEEM.

MED (2005). La evaluación de los aprendizajes de los estudiantes en la educación

MED (2009). Diseño curricular nacional de educación básica regular. Lima: Ministerio de Educación.

MED. (2009). Guía de Evaluación para la Educación Técnico Productiva. Lima: Ministerio de Educación.

MINEDU (2012). Marco del buen desempeño docente. Lima: Corporación Gráfica Navarrete.

MINEDU (2016). Currículo Nacional: Educación Básica Regular - Programa Curricular de Educación Secundaria. Lima: Ministerio de Educación.

Rossi, P.H., \& Freeman, H.E. (1993). Evaluación. Un enfoque sistemático para programas sociales. México, D.F.: Trillas.

Rueda Beltran, M. (2009). La Evaluación del Desempeño Docente: Consideraciones desde el enfoque por competencias. REDALYC Red de Revistas Científicas de América Latina, el Caribe, España y Portugal - México., 07 - 12.

Torres Arias, R. (2013). La Evaluación Formativa. San José: Ministerio de Educación Pública.

Supo, José (2014). Cómo probar una hipótesis. Lima: Bioestadistico.

\section{CORRESPONDENCIA:}

Mag. Alberto Huamani Gallo

lubeto33@gmail.com 